\title{
Josephson current in ballistic superconductor-graphene systems
}

\author{
Imre Hagymási, ${ }^{1}$ Andor Kormányos, ${ }^{2}$ and József Cserti ${ }^{1}$ \\ ${ }^{1}$ Department of Physics of Complex Systems, Eötvös University, Pázmány Péter sétány 1/A, H-1117 Budapest, Hungary \\ ${ }^{2}$ Department of Physics, Lancaster University, Lancaster LA1 4YB, United Kingdom
}

(Received 22 June 2010; published 12 October 2010)

\begin{abstract}
We calculate the phase, the temperature and the junction length dependence of the supercurrent for ballistic graphene Josephson junctions. For low temperatures we find nonsinusoidal dependence of the supercurrent on the superconductor phase difference for both short and long junctions. The skewness, which characterizes the deviaton of the current-phase relation from a simple sinusoidal one, shows a linear dependence on the critical current for small currents. We discuss the similarities and differences with respect to the classical theory of Josephson junctions, where the weak link is formed by a diffusive or ballistic metal. The relation to other recent theoretical results on graphene Josephson junctions is pointed out and the possible experimental relevance of our work is considered as well.
\end{abstract}

DOI: 10.1103/PhysRevB.82.134516

PACS number(s): 74.45.+c, 74.50.+r, 74.78.Na, 03.65.Sq

\section{INTRODUCTION}

The peculiar electronic properties of graphene first observed experimentally by Geim et al. ${ }^{1}$ and Zhang et al. ${ }^{2}$ can accurately be described by massless two-dimensional Dirac fermion excitations (for reviews on the physics of graphene see, e.g, Refs. 3-6).

Owing to the proximity effect a superconductor can induce nonzero pair potential in the graphene as well. ${ }^{7}$ Such graphene-superconductor hybrid structures and in particular the Andreev-reflection taking place at the graphenesuperconductor interface was first studied theoretically by Beenakker ${ }^{8}$ (for a review on Andreev reflection in graphene see Ref. 9). Soon after Beenakker's pioneering work, supercurrent between two superconducting electrodes on top of a graphene monolayer has been observed experimentally by Heersche et al. $^{10}$ and later in Refs. 11-15. In particular, the experimental results of Ref. 12 attest to the ballistic propagation of quasiparticles in graphene-superconductor hybrid structures, whereas the experiment of Du et al. ${ }^{14}$ gave evidence that it was possible to fabricate transparent superconductor-graphene (SG) interfaces. These experiments have also sparked considerable theoretical interest in superconductor-graphene-superconductor (SGS) heterostructures. The short junction limit, where the coherence length $\xi=\hbar v_{F} / \Delta_{0}$ (here $v_{F}$ is the graphene Fermi velocity and $\Delta_{0}$ is the superconducting gap) is smaller than the length $L$ of the junction, was first studied by Titov and Beenakker ${ }^{16}$ assuming ballistic graphene. In the opposite, long junction limit the density of states of the Andreev levels was calculated first by Titov, Ossipov, and Beenakker. ${ }^{17}$ Subsequently, numerous other theoretical works investigated the Josephson current in SGS structures. ${ }^{14,18-23}$ The tunneling effect in SG structures has been studied in several works ${ }^{24-28}$ as well. Other works in the field of graphene-superconductor heterostructures include studies on crossed Andreev reflection in a graphene bipolar transistor, ${ }^{29,30}$ on $s$ - and $d$-wave SG junctions ${ }^{31,32}$ and on ferromagnetic SG structures. ${ }^{33,34}$ Very recently, using a phase-sensitive superconducting quantum interference device interferometry technique Chialvo et al. ${ }^{35}$ has studied experimentally the current-phase relation $(C \Phi R)$ of graphene Josephson junctions.
In this work we calculate the Josephson current in SGS structure as a function of the superconductor phase difference, the temperature, and the length of the junction. In our theoretical treatment we adapted the method used by Brouwer and Beenakker for metallic chaotic Josephson junctions. ${ }^{36}$ The approach allows to obtain results for finite temperature and is valid for junctions of arbitrary length. We note that this method has already been applied for calculating the persistent current through a $n-p$ junction in graphene. ${ }^{21}$ Wherever possible, we compare our results to previous ones derived for superconductor-normal conductorsuperconductor (SNS) junctions, where the normal conductor is a ballistic metal.

The rest of the paper is organized in the following way: in the next section we introduce the theoretical approach that we use to obtain the Josephson current. In Sec. III we present and discuss the results of numerical calculations. Finally, in Sec. IV we give a brief summary.

\section{THEORETICAL CONCEPT}

We consider a Josephson junction in the $x-y$ plane. The normal graphene region $(G)$ at $|x|<L / 2$ separates the two superconducting regions formed by covering the graphene layer by two superconducting electrodes $(S)$ in the regions $x<-L / 2$ and $x>L / 2$ (for the geometry see Ref. 16). The width of the Josephson junction along the $y$ axis is $W$. Owing to the valley degeneracy of the Hamiltonian, the full DiracBoguliubov-de Gennes (DBdG) equations for graphenesuperconductor systems decouple to two four by four, reduced Hamiltonians that are related to each other by a unitary transformation (see, e.g., Ref. 8). We now take the one corresponding to the valley $\mathbf{K}$. Then the quasiparticle excitations in the SGS systems are described by the reduced $\mathrm{DBdG}$ equations

$$
\left(\begin{array}{cc}
H_{0}-\mu & \Delta(x, y) \\
\Delta^{*}(x, y) & \mu-H_{0}
\end{array}\right) \Psi=\varepsilon \Psi,
$$

where $H_{0}=-i \hbar v_{F}\left(\sigma_{x} \partial_{x}+\sigma_{y} \partial_{y}\right)+U(x, y) \sigma_{0}$ is the Dirac Hamiltonian. Here $\sigma_{x}$ and $\sigma_{y}$ are Pauli matrices, $\sigma_{0}$ is the unit 
matrix, $\mu$ is the chemical potential, and $\varepsilon>0$ is the excitation energy. The superconductor electrodes are doped by the potential $U(x, y)=U_{0} \Theta(|x|-L / 2)$ (here $U_{0}<0$ and constant, and $\Theta(x)$ is the Heaviside function). The wave function $\Psi$ $=\left(\Psi_{e}, \Psi_{h}\right)^{T}$ is comprised of electron $\Psi_{e}$ and hole $\Psi_{h}$ wave functions which have opposite spin and valley indices. For the pair potential $\Delta(x, y)$ we assume a simple model: its magnitude $\Delta_{0}$ is constant in the $S$ regions, changes step functionlike at the SG interfaces (so called "rigid boundary condition", see Ref. 37) and is zero in the normal conducting region. Similarly, we assume that its phase is piecewise constant in the $S$ regions. Hence, the pair potential is given by $\Delta(x, y)=\Delta_{0} e^{-i \phi / 2}$ for $x<-L / 2$ and $\Delta(x, y)=\Delta_{0} e^{i \phi / 2}$ for $x$ $>L / 2$. Band bending or other effects of the superconducting electrodes are neglected (see, e.g., Ref. 38 for the discussions of some of these effects in the case of normal conducting metal electrodes).

The Josephson current at finite temperature is given by ${ }^{36}$

$$
I=-2 k_{\mathrm{B}} T \frac{4 e}{\hbar} \frac{d}{d \phi} \int_{0}^{\infty} d \varepsilon \varrho(\varepsilon) \ln \left[2 \cosh \left(\frac{\varepsilon}{2 k_{\mathrm{B}} T}\right)\right],
$$

where $\phi$ is the phase difference across the junction, $\varrho(\varepsilon)$ is the density of states of the Andreev levels. The factor of 4 accounts for the spin and valley degeneracies. As one can see from Eq. (2), a necessary step to calculate the Josephson current is to obtain the density of states of Andreev bound states in the SGS junction. To this end one can in principle proceed in the following way: one can write down a trial wave function in all three regions of the SGS structure. The boundary conditions at the two graphene-superconductor boundaries of the SGS junction then result in a secular equation $\mathcal{F}(\varepsilon)=0$ whose solutions $\varepsilon_{i}$ give the energies of the Andreev bound states. For finite $U_{0}$ we obtained a $8 \times 8$ determinant for the secular equation (since this determinant is quite lengthy here we do not present its detailed form). Once the energy levels $\varepsilon_{i}$ of the SGS junction are known, the density of state is given by $\varrho(\varepsilon)=\Sigma_{i} \delta\left(\varepsilon-\varepsilon_{i}\right)$.

However, the above outlined method is numerically quite cumbersome since one has to search for the zeros of the secular equation $\mathcal{F}(\varepsilon)=0$. To overcome this problem we now follow the method used by Brouwer and Beenakker in Ref. 36. They rewrote the expression for the Josephson current given in Eq. (2) in a more convenient form. Here we only summarize the main steps of the derivation. The secular equation can be written as $\mathcal{F}(\varepsilon)=\mathcal{F}_{0} \Pi_{i}\left[\left(\varepsilon-\varepsilon_{i}\right)\right]=0$, where $\mathcal{F}_{0}$ is a function of $\varepsilon$ but does not have zeros in the complex plane. Thus it is easy to see that the density of states can be expressed as

$$
\varrho(\varepsilon)=-\frac{1}{\pi} \frac{d}{d \varepsilon} \operatorname{Im} \ln \mathcal{F}\left(\varepsilon+i 0^{+}\right),
$$

where $0^{+}$is a positive infinitesimal. Using the analytic properties of $\mathcal{F}$ in the upper half of the complex $\varepsilon$ plane and the fact that under the change $\varepsilon \rightarrow-\varepsilon$ the function $\mathcal{F}$ goes over into its complex conjugate (physically, this follows from the electron-hole symmetry), the $\varepsilon$ integration can be extended from $-\infty$ to $\infty$. Finally, after performing a partial integration the Josephson current in Eq. (2) can be rewritten as

$$
I=-\frac{2 e}{i \pi \hbar} \frac{d}{d \phi} \int_{-\infty+i 0^{+}}^{\infty+i 0^{+}} d \varepsilon \tanh \left(\frac{\varepsilon}{2 k_{\mathrm{B}} T}\right) \ln \mathcal{F}(\varepsilon) .
$$

Now closing the integration contour in the upper half of the complex $\varepsilon$ plane and applying the residue theorem the Josephson current becomes

$$
I=-\frac{4 e}{\hbar} 2 k_{\mathrm{B}} T \sum_{n=0}^{\infty} \frac{d}{d \phi} \ln \mathcal{F}\left(i \omega_{n}\right)
$$

where $i \omega_{n}=i(2 n+1) \pi k_{\mathrm{B}} T$ are Matsubara frequencies. Note that in our model $\ln \mathcal{F}(\varepsilon)$ has no singularities for $\operatorname{Im} \varepsilon>0$, thus the poles of the integrand in Eq. (4) come only from the hyperbolic tangent function. The main advantage of this result is that one does not need to obtain explicitly the solutions of the secular equation $\mathcal{F}(\varepsilon)=0$. Moreover, this method immediately gives the finite temperature dependence of the Josephson current. In the numerical calculations it turns out that the sum in Eq. (5) is rapidly convergent and usually one need to include only a finite number of terms. A similar result has been found for the persistent current through a $n-p$ junction in graphene. ${ }^{21}$

We now consider the experimentally relevant case of highly doped superconductor electrodes, i.e., the limit $U_{0}$ $\rightarrow-\infty$. By matching the wave functions at the graphenesuperconductor boundaries of the SGS structure we found the same secular equation $\mathcal{F}\left(\varepsilon, q_{m}\right)=0$ as that obtained by Titov and Beenakker using the transfer matrix method [see Eq. (14) in Ref. 16]. We used the "infinite mass" boundary conditions $^{39}$ at $y=0$ and $y=W$ for which $q_{m}=(m+1 / 2) \pi / W$, where $m=0,1,2, \ldots$ (for $W \gg L$ the choice of the boundary conditions is irrelevant). For a given $m$ the solutions of the quantization condition $\mathcal{F}\left(\varepsilon, q_{m}\right)=0$ give the Andreev energy levels $\varepsilon_{m}$ for $\varepsilon_{m}<|\Delta|$. The secular equation $\mathcal{F}\left(\varepsilon, q_{m}\right)=0$ is valid both in short and long junction limit. ${ }^{16}$ One can show that $\mathcal{F}\left(-\varepsilon+i 0^{+}, q\right)=\mathcal{F}^{*}\left(\varepsilon+i 0^{+}, q\right)$ which is a necessary condition $^{36}$ for writing the Josephson current in the form of Eq. (4).

The current contribution from each propagating mode with transverse momentum $q_{m}$ can be calculated separately and the total current is the sum over these contributions

$$
I=-\frac{4 e}{\hbar} 2 k_{\mathrm{B}} T \sum_{m=0}^{M} \sum_{n=0}^{\infty} \frac{d}{d \phi} \ln \mathcal{F}\left(i \omega_{n}, q_{m}\right),
$$

where $M$ is the number of propagating modes and the function $\mathcal{F}(\varepsilon, q)$ determines the energy levels for a given transverse momentum $q$. This equation is our starting point for calculating the supercurrent through a graphene based Josephson junction.

Further analytical progress can be made in the short junction limit $(L \ll \xi)$ because the Andreev levels $\varepsilon_{m}$ can in that case be obtained in a closed form [see Eq. (16) in Ref. 16]. Similarly, the summation over the Matsubara frequencies in Eq. (6) can be performed analytically using the identity $\sum_{k=0}^{\infty} 1 /\left[(2 k+1)^{2}+x^{2}\right]=\pi \tanh (\pi x / 2) /(4 x)$ (see Ref. 40) and we find 


$$
I=\frac{e \Delta_{0}^{2}(T)}{\hbar} \sin \phi \sum_{m=0}^{\infty} \frac{\tau_{m}}{\varepsilon_{m}} \tanh \left(\frac{\varepsilon_{m}}{2 k_{\mathrm{B}} T}\right) .
$$

Here $\varepsilon_{m}$ and $\tau_{m}$ can be found in Ref. 16 while the temperature dependence of the superconductor gap $|\Delta|=\Delta_{0}(T)$ for $s$-wave superconductors is given by

$$
\ln \frac{\Delta_{0}(0)}{\Delta_{0}(T)}=2 \sum_{n=1}^{\infty}(-1)^{n+1} K_{0}\left[n \frac{\Delta_{0}(T)}{k_{\mathrm{B}} T}\right],
$$

where $K_{0}(x)$ is the zero order modified Bessel function, $\Delta_{0}(0)=\left(e^{\gamma} / \pi\right) k_{\mathrm{B}} T_{c}=0.567 k_{\mathrm{B}} T_{c}$ and $\gamma$ is the Euler's constant. ${ }^{41}$ For zero temperature from Eq. (7) one can arrive at the same expression for the Josephson current as that obtained by Titov and Beenakker (Ref. 16). For finite temperatures the summation over the transversal modes $m$ in Eq. (6) cannot be evaluated analytically but numerically can easily be treated.

\section{NUMERICAL RESULTS}

We now present the results of numerical calculations for the Josephson current using the most general formula given by Eq. (6). Figure 1 shows the supercurrent as a function of phase $\phi$ for a number of interesting case: for short $(L \ll \xi)$, intermediate $(L \simeq \xi)$ and long $(L \gg \xi)$ junctions, assuming $\mu$ $=0$ and for finite $\mu / \Delta_{0}$ as well. One can make the following general observations: (a) the maximum current increases by increasing the doping $\left(\mu / \Delta_{0}\right.$ value) and by decreasing the temperature or the junction length; and (b) at higher temperatures the current shows a simple sinusoidal dependence on the phase in all of the cases while at low temperatures the position of the maxima of the currents are shifted to the right resulting in a skewness of the curves. Following Ref. 35 we define the skewness by $S=2 \phi_{\max } / \pi-1$, where $\phi_{\max }$ is the position of the maxima of the supercurrent. Both the tendency to simple harmonic dependence for $T \rightarrow T_{c}$ and a positive skewness (i.e., $\phi_{\max }>\pi / 2$ ) are in line with previous calculations on Josephson current in weak links which comprise a normal conducting metal or a tunnel barrier and assume that the pair potential changes abruptly at the normalsuperconductor interface (see, e.g., Ref. 37 for a review).

We find especially interesting the results shown in Figs. $1(\mathrm{e})$ and 1(f). In the long junction limit for $\mu=0$ we find that the skewness is very small even at $T / T_{c}=0$ [the curve denoted by black squares in Fig. 1(e)] thus the C $\Phi$ R resembles a harmonic dependence. In contrast, still in the long junction limit but for $\mu / \Delta_{0}=20$ and $T / T_{c}=0$ [black squares in Fig. $1(\mathrm{f})]$ we see that the current depends almost linearly on the phase and the curve resembles a sawtooth. [The transition to a sawtoothlike dependence can already be seen in Fig. 1(d) where $L / \xi=1.1$, cf. Fig. 1(b) showing the short junction limit.] It is interesting to note that the theoretical result for clean, long SNS junctions at low temperature is a sawtoothed CФR. ${ }^{37,42}$ Our numerics suggests that for SGS junctions in the same limit the sawtooth is somewhat rounded-off and the slope of the curve is finite when $\phi \rightarrow \pi$. Thus, the CФR in long, clean SGS junction seems to be closely resembling of but not identical to the corresponding case in SNS junctions.
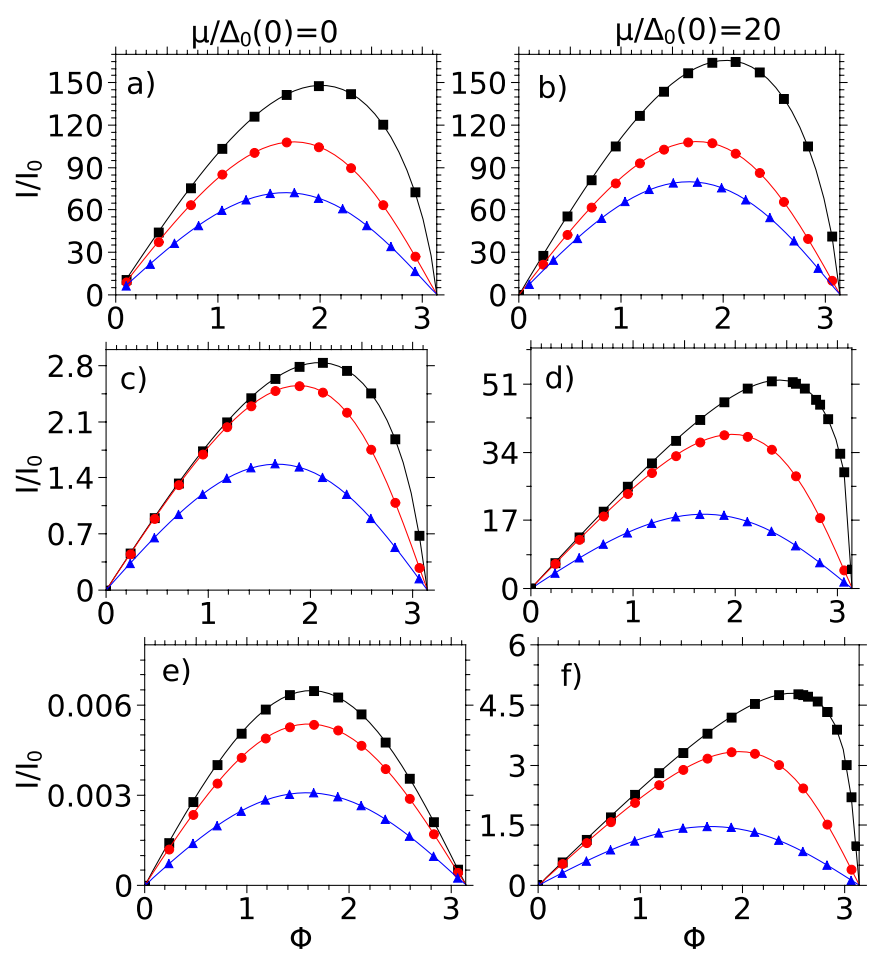

FIG. 1. (Color online) The supercurrent [in units of $I_{0}$ $\left.=e \Delta_{0}(0) / \hbar\right]$ as a function of the phase difference $\phi$. The parameters are as follows: In (a) and (b) (short junction limit) $\xi / L=20$ and $T / T_{c}=0,0.53,0.71$ (black $\square$, red $\bigcirc$, and blue $\triangle$, respectively). In (c) and (d) (here $L \simeq \xi$ ) we used $\xi / L=0.91$ and $T / T_{c}=0,0.18,0.35$ (black $\square$, red $\bigcirc$, and blue $\triangle$, respectively). In (e) and (f) we consider long junctions $(\xi / L=0.05)$. In (e) $T / T_{c}=0,0.035,0.053$ (black $\square$, red $\bigcirc$, and blue $\triangle$, respectively). In (f) $T / T_{c}=0,0.018,0.035$ (black $\square$, red $\bigcirc$, and blue $\triangle$, respectively). The chemical potential is $\mu=0$ in (a), (c), and (e), whereas it is $\mu / \Delta_{0}=20$ in (b), (d), and (f). The width of the junction is $\xi / W=0.05$ in all cases.

Further analytical progress would be needed to clarify this point.

We calculated the skewness $S$ as a function of the critical supercurrent $I_{c}$ (the value of the current at $\phi_{\max }$ ) and plotted the results for three different $\xi$ values while keeping the junction length $L$ and width $W$ constant. The used $\xi / L$ values go from $\xi / L=0.35$ (long junction limit) to $\xi / L=1.75$ (short junction limit). There are two important things to notice in Fig. 2 for small critical currents: a) for a given junction length $L$, as $I_{c} \rightarrow 0$ (for higher temperatures) the skewness $S$ also goes to zero, i.e., the CФR is approaching a simple sinusoidal form and (b) $S$ depends linearly on $I_{c}$ for small critical currents while at larger $I_{c}$ the dependence clearly deviates from a simple linear relation. The skewness has recently been measured in Ref. 35 and the case of $\xi / L=0.35$, $\xi / W=0.0077$, shown by black dots in Fig. 2, in principle, corresponds to that of sample $\mathrm{B}$ in this experiment [with estimated coherence length of $\xi \approx 100 \mathrm{~nm}$ (Ref. 43)]. Our calculations give a smaller slope than the measurements in Ref. 35. According to our numerics, the larger slopes observed in this experiment would be attainable in the short junction limit. Note however, that the exact slope would also depend on the value of the chemical potential which was not known, and importantly, the samples in the experiment of 


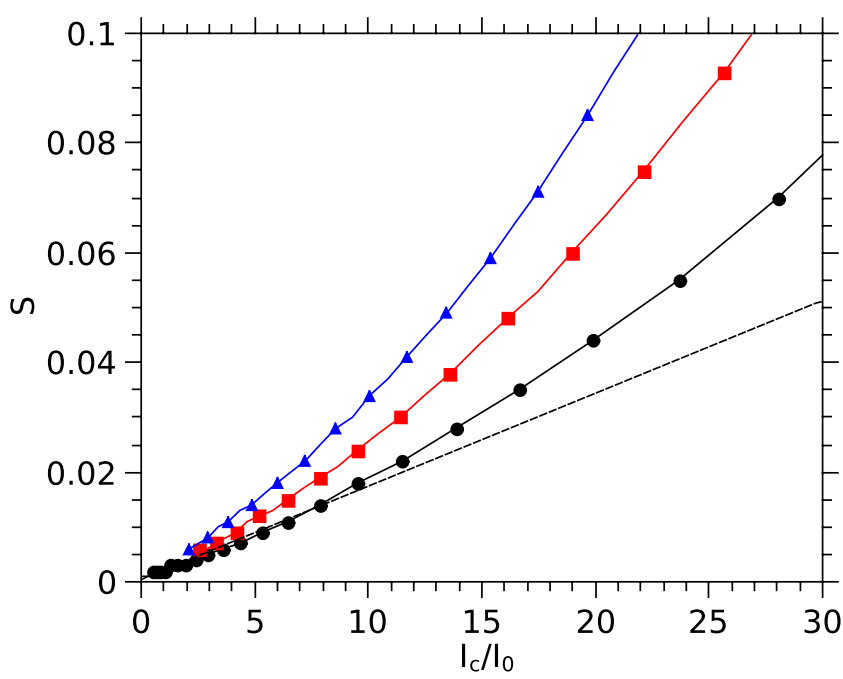

FIG. 2. (Color online) The skewness $S$ as a function of the critical current $I_{c}$ for different coherence lengths $\xi$. The parameters are $\xi / L=0.35, \xi / W=0.0077$ (black dots), $\xi / L=1.05, \xi / W=0.0231$ (red squares), and $\xi / L=1.75, \xi / W=0.0385$ (blue triangles). The lines are guides to the eye. The ratio of the chemical potential and the superconducting gap was $\mu / \Delta_{0}=10$. The dotted line shows that for small critical currents the skewness depends linearly on $I_{c}$.

Ref. 35 are likely to have been in the quasidiffusive limit, therefore we cannot expect quantitative agreement with our ballistic theory.

We have also calculated the temperature dependence of the critical current for short, intermediate and long junctions,

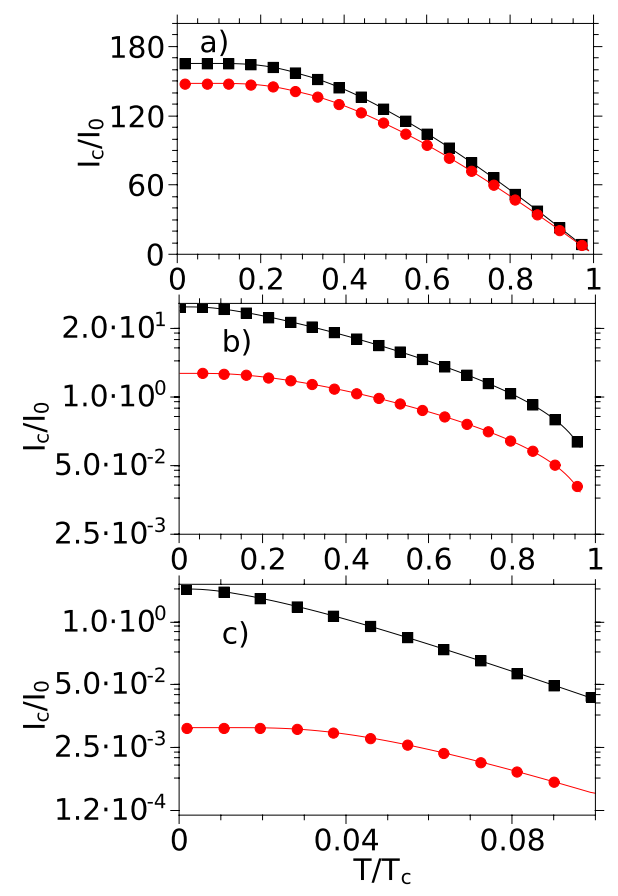

FIG. 3. (Color online) The critical current $I_{c}$ as a function of $T / T_{c}$. The parameters are $\xi / L=20$ in (a), $\xi / L=0.91$ in (b), and $\xi / L=0.05$ in (c). In (b) and (c) we used logarithmic scale. Red $\bigcirc$ denote the results for $\mu=0$ and black $\square$ for $\mu / \Delta_{0}(0)=20$. The width of the sample was fixed: $\xi / W=0.05$.

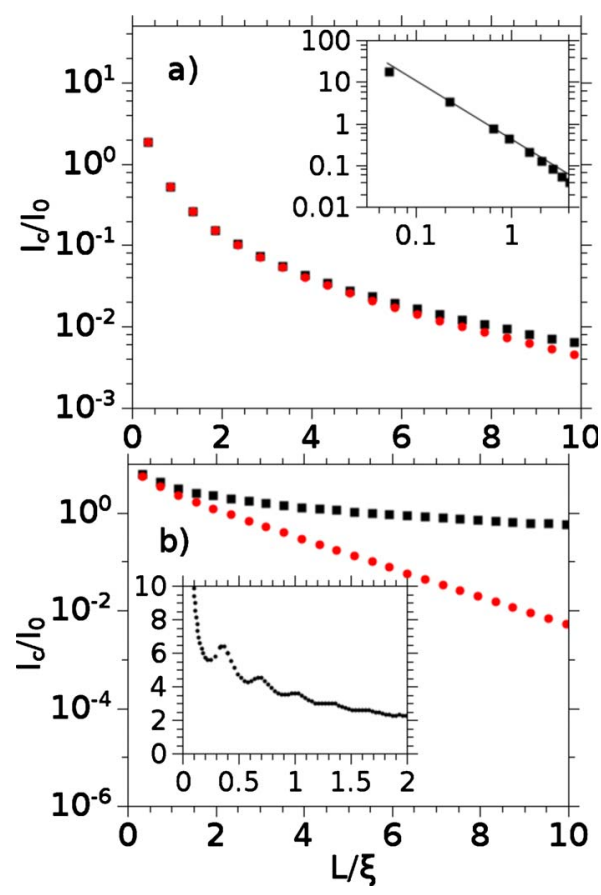

FIG. 4. (Color online) The critical current $I_{c}$ as a function of the junction length $L / \xi$ in logarithmic scale. In (a) we used $\mu=0$. Black $\square$ and red $\bigcirc$ denote the results of $T / T_{c}=0.0$ and $T / T_{c}=0.06$ calculations, respectively. The symbols in the inset of (a) show $I_{c}$ for $T / T_{c}=0.0, L / \xi<1$ in double logarithmic plot, along with the fitted linear function (solid line, see text). In (b) the chemical potential is $\mu / \Delta_{0}(0)=10$. Black $\square$ and red $\bigcirc$ denote the results of $T / T_{c}=0.0$ and $T / T_{c}=0.18$ calculations, respectively. The inset of (b) shows the $T / T_{c}=0.0$ calculations in linear scale for $L / \xi \leq 2$. The width of the junction was $\xi / W=0.05$ in all cases.

taking two values of the chemical potential $\mu$. The temperature dependence of the pair potential was taken into account using Eq. (8). The results are shown in Fig. 3. At this point it is interesting to make a quick sidestep and note that Titov and Beenakker (Ref. 16) showed that for short junctions, at zero temperature and at the Dirac point the C $\Phi \mathrm{R}$ for ballistic graphene is formally identical to the classical result of Kulik and Omel'yanchuk, ${ }^{44}$ which, however, assumes diffusive normal metal as a weak link. Looking at the $I_{c}-T$ curves in the short junction limit [Fig. 3(a)] one can see that they are also qualitatively similar to the corresponding result of Kulik and Omel'yanchuk ${ }^{44}$ (cf. Fig. 7 in Ref. 37). The close resemblance of certain properties the two types of Josephson junctions therefore seems to extend to the temperature dependence of the critical current as well. In the opposite, long junction regime, for $\mu=0$ [shown by red circles in Fig. 3(c)] one can observe a short plateau in the current for small temperatures followed by an exponential decay. Interestingly, a qualitatively very similar result has been obtained by González and Perfetto in Ref. 22, assuming tunnel coupling between the superconductor and the graphene and using a different formalism than ours. In the case of finite doping [black squares in Fig. 3(c)] an exponential decay of $I_{c}$ can be seen basically in the whole temperature range. The $I_{c}$ in clean SNS junction exhibits the same qualitative dependence on $T$ (Ref. 37). 
Experimentally, the $I_{c}-T$ relation was measured by Du et al. (Ref. 14) and by Ojeda-Aristizabal et al. (Ref. 15). Again, quantitative comparison with our results is not possible because the graphene samples in these experiments were in the diffusive limit, but the observed dependence of $I_{c}$ on the temperature was qualitatively similar to the results shown in Fig. 3(a).

Finally, we studied the length dependence of the critical current and the results are shown in Fig. 4. At the Dirac point $(\mu=0)$ one can observe an exponential decay of $I_{c}$ for $L / \xi$ $\gg 1$ [main panel of Fig. 4(a)]. We could not see the $\sim 1 / L^{2}$ dependence predicted in Ref. 22. For $L / \xi \lesssim 1$ and $T / T_{c}=0$, however, we do find a power-law dependence $I_{c} \sim L^{b}$ [see the inset of Fig. 4(a)] and fitting the numerical results we obtained $b=-1.4$. This is remarkably close to the results of the self-consistent tight-binding calculations of Black-Schaffer and Doniach (Ref. 23) who found $b=-1.3$. Considering now the case of doped graphene weak link, we found that for $L / \xi>1$ the $L$ dependence of $I_{c}$ can be well fitted by $I_{c} / I_{0}$ $=I_{a} e^{-b L / \xi}$ with $b \approx 1$. Exponentially small critical current is also typical for clean SNS junctions if $L$ is larger than the thermal coherence length (for details see, e.g., Ref. 37). We note that in the case of $L / \xi<1$ and $T / T_{c}=0$ one can see oscillations in the $I_{c}$ vs $L$ curve [shown in the inset of Fig. 4(b)] whose study is left to a future work.

\section{SUMMARY}

In this work we calculated the Josephson current in ballistic SGS structures. The most important assumption we made is that one can use rigid boundary conditions. i.e., that the change of the pair potential is step functionlike at the SG interfaces and that it does not depend on the supercurrent. We developed a general and numerically efficient approach to obtain the current-phase relation for arbitrary length of the junction as well as for finite doping and temperature. At low temperatures we have found that the current-phase relation differs from a simple harmonic dependence. In the case of short junctions and small critical currents the deviation of the current-phase curve from the sinusoidal form, the skewness, shows a linear dependence on the critical current, similarly to the observation of a recent experiment, ${ }^{35}$ though the slope of the curve did not match the experimental one. This is likely to be due to the fact that in the experiment the graphene sample was quasidiffusive. In the long junction limit our results show that in clean SGS junctions the current-phase relation transforms from the sinusoidal form at $T \lesssim T_{c}$ to a curve resembling sawtooth at $T \ll T_{c}$. We have also calculated the temperature and junction length dependence of the critical current. We have found similarities to both classical results for SNS junctions and recent ones obtained for graphene but using a different formalism. ${ }^{22}$ In respect of these numerical calculations further theoretical progress is needed to unravel the relation between the SGS and SNS results.

Since the fabrication of both ballistic graphene samples and transparent SG interfaces have already been demonstrated experimentally, ${ }^{12,14}$ we believe that our theoretical approach may be useful in the future to understand and analyze experimental data. In particular, the measurement of the $\mathrm{C} \Phi \mathrm{R}$ and the length, and temperature dependence of $I_{c}$ in short junctions should be feasible.

Note added. During the peer-review process of the manuscript, a relevant preprint has appeared, ${ }^{45}$ where the authors study the temperature dependence of the Josephson current using self-consistent tight-binding numerical computations. Their work is thus complementary to ours and can help to understand the scope of certain approximations, e.g., the rigid-boundary condition that we employed.

\section{ACKNOWLEDGMENTS}

Supported by the Marie Curie ITN project NanoCTM (Grant No. FP7-PEOPLE-ITN-2008-234970) and the Hungarian Science Foundation OTKA under Contracts No. 75529 and No. 81492. A.K. also acknowledges the support of EPSRC. We acknowledge fruitful discussions with C. W. J. Beenakker, E. Perfetto, N. Mason, D. J. Van Harlingen, and C. Chialvo.
${ }^{1}$ K. Novoselov et al., Science 306, 666 (2004).

${ }^{2}$ Y. Zhang, Y. Tan, H. Stormer, and P. Kim, Nature (London) 438 , 201 (2005).

${ }^{3}$ M. I. Katsnelson, Mater. Today 10, 20 (2007).

${ }^{4}$ M. I. Katsnelson and K. S. Novoselov, Solid State Commun. 143, 3 (2007).

${ }^{5}$ A. K. Geim and K. S. Novoselov, Nature Mater. 6, 183 (2007).

${ }^{6}$ A. H. C. Neto, F. Guinea, N. M. R. Peres, K. S. Novoselov, and A. K. Geim, Rev. Mod. Phys. 81, 109 (2009).

${ }^{7}$ G. Fagas, G. Tkachov, A. Pfund, and K. Richter, Phys. Rev. B 71, 224510 (2005).

${ }^{8}$ C. W. J. Beenakker, Phys. Rev. Lett. 97, 067007 (2006).

${ }^{9}$ C. W. J. Beenakker, Rev. Mod. Phys. 80, 1337 (2008).

${ }^{10}$ H. B. Heersche, P. Jarillo-Herrero, J. B. Oostinga1, L. M. K. Vandersypen, and A. F. Morpurgo, Nature (London) 446, 56
(2007).

${ }^{11}$ H. B. Heersche, P. Jarillo-Herrero, J. B. Oostinga1, L. M. K. Vandersypen, and A. F. Morpurgo, Solid State Commun. 143, 72 (2007); H. B. Heersche, P. Jarillo-Herrero, J. B. Oostinga, L. M. K. Vandersypen, and A. F. Morpurgo, Graphene Week 2006 International Conference, Dresden, Germany, 2006 Eur. Phys. J. Spec. Top. 148, 27 (2007)].

${ }^{12}$ F. Miao, S. Wijeratne, Y. Zhang, U. C. Coskun, W. Bao, and C. N. Lau, Science 317, 1530 (2007).

${ }^{13}$ A. Shailos, W. Nativel, A. Kasumov, C. Collet, M. Ferrier, S. Guéron, R. Deblock, and H. Bouchiat, EPL 79, 57008 (2007).

${ }^{14}$ X. Du, I. Skachko, and E. Y. Andrei, Phys. Rev. B 77, 184507 (2008).

${ }^{15}$ C. Ojeda-Aristizabal, M. Ferrier, S. Guéron, and H. Bouchiat, Phys. Rev. B 79, 165436 (2009). 
${ }^{16}$ M. Titov and C. W. J. Beenakker, Phys. Rev. B 74, 041401(R) (2006).

${ }^{17}$ M. Titov, A. Ossipov, and C. W. J. Beenakker, Phys. Rev. B 75, 045417 (2007).

${ }^{18}$ A. G. Moghaddam and M. Zareyan, Phys. Rev. B 74, 241403(R) (2006).

${ }^{19}$ A. G. Moghaddam and M. Zareyan, Appl. Phys. A: Mater. Sci. Process. 89, 579 (2007).

${ }^{20}$ M. Maiti and K. Sengupta, Phys. Rev. B 76, 054513 (2007).

${ }^{21}$ C. W. J. Beenakker, A. R. Akhmerov, P. Recher, and J. Tworzydło, Phys. Rev. B 77, 075409 (2008).

${ }^{22}$ J. González and E. Perfetto, Phys. Rev. B 76, 155404 (2007); J. Phys.: Condens. Matter 20, 145218 (2008).

${ }^{23}$ A. M. Black-Schaffer and S. Doniach, Phys. Rev. B 78, 024504 (2008).

${ }^{24}$ P. Burset, A. L. Yeyati, and A. Martín-Rodero, Phys. Rev. B 77, 205425 (2008).

${ }^{25}$ D. Rainis, F. Taddei, F. Dolcini, M. Polini, and R. Fazio, Phys. Rev. B 79, 115131 (2009).

${ }^{26}$ S. Bhattacharjee and K. Sengupta, Phys. Rev. Lett. 97, 217001 (2006).

${ }^{27}$ S. Bhattacharjee, M. Maiti, and K. Sengupta, Phys. Rev. B 76, 184514 (2007).

${ }^{28}$ J. C. Cuevas and A. L. Yeyati, Phys. Rev. B 74, 180501(R) (2006).

${ }^{29}$ J. Cayssol, Phys. Rev. Lett. 100, 147001 (2008).

${ }^{30}$ J. Linder, M. Zareyan, and A. Sudbø, Phys. Rev. B 80, 014513
(2009).

${ }^{31}$ J. Linder and A. Sudbø, Phys. Rev. Lett. 99, 147001 (2007).

${ }^{32}$ J. Linder and A. Sudbø, Phys. Rev. B 77, 064507 (2008).

${ }^{33}$ A. G. Moghaddam and M. Zareyan, Phys. Rev. B 78, 115413 (2008).

${ }^{34}$ Y. Asano, T. Yoshida, Y. Tanaka, and A. A. Golubov, Phys. Rev. B 78, 014514 (2008).

${ }^{35}$ C. Chialvo, I. C. Moraru, D. J. V. Harlingen, and N. Mason, arXiv:1005.2630v1 (unpublished).

${ }^{36} \mathrm{P}$. W. Brouwer and C. W. J. Beenakker, Chaos, Solitons Fractals 8, 1249 (1997).

${ }^{37}$ A. A. Golubov, M. Yu. Kupriyanov, and E. Ilichev, Rev. Mod. Phys. 76, 411 (2004).

${ }^{38}$ P. A. Khomyakov, A. A. Starikov, G. Brocks, and P. J. Kelly, Phys. Rev. B 82, 115437 (2010).

${ }^{39}$ J. Tworzydło, B. Trauzettel, M. Titov, A. Rycerz, and C. W. J. Beenakker, Phys. Rev. Lett. 96, 246802 (2006).

${ }^{40}$ I. S. Gradshteyn and I. M. Ryzhik, Table of Integrals, Series, and Products, 5th ed. (Academic Press, London, UK, 1994).

${ }^{41}$ A. A. Abrikosov, L. P. Gor'kov, and I. Y. Dzyaloshinskii, Quantum Field Theoretical Methods in Statistical Physics, 2nd ed. (Pergamon Press, Oxford, London, UK, 1965).

${ }^{42}$ C. Ishii, Prog. Theor. Phys. 44, 1525 (1970).

${ }^{43}$ N. Mason (private communication).

${ }^{44}$ I. O. Kulik and A. N. Omelyanchuk, JETP Lett. 21, 96 (1975).

${ }^{45}$ A. M. Black-Schaffer and J. Linder, arXiv:1008.2619 (unpublished). 